\title{
QUASILINEAR DIRICHLET PROBLEM IN A PERIODICALLY PERFORATED DOMAIN
}

\author{
L. Boukrim, A. Hakim and T. MekKaoui
}

Université Moulay Ismail and Université Cadi Ayyad, Morocco

Abstract. Consider the following quasilinear Dirichlet problem

$$
\left\{\begin{array}{lll}
-\Delta_{p} u_{\varepsilon}=f_{\varepsilon} & \text { in } & \Omega_{\varepsilon}=\Omega \backslash T_{\varepsilon} \\
u_{\varepsilon}=0 & \text { on } & \partial \Omega_{\varepsilon}
\end{array}\right.
$$

where $1<p \leq 2,\left(f_{\varepsilon}\right)$ a sequence of functions in $L^{p^{\prime}}(\Omega)$ such that $f_{\varepsilon} \rightarrow f$ in $L^{p^{\prime}}(\Omega)$ where $p^{\prime}$ is the conjugate of $p, \Omega$ is a bounded domain in $\mathbb{R}^{N}$ $(N \geq 2)$ and $T_{\varepsilon}$ the union of inclusions contained in $\Omega$ which are $\varepsilon$ periodically distributed. In this paper we give the limit problem and errors estimates for the problem $\left(P_{\varepsilon}\right)$.

\section{INTRODUCTION}

We consider here the homogenization of the following quasilinear Dirichlet problem

$$
\left\{\begin{array}{cll}
-\Delta_{p} u_{\varepsilon}=f_{\varepsilon} & \text { in } & \Omega_{\varepsilon}=\Omega \backslash T_{\varepsilon} \\
u_{\varepsilon}=0 & \text { on } & \partial \Omega_{\varepsilon}
\end{array}\right.
$$

where,

- $\Delta_{p} u=\operatorname{div}\left(|\nabla u|^{p-2} \nabla u\right)$

- $1<p \leq 2$

- $\left(f_{\varepsilon}\right)$ is a convergent sequence of functions to $f$ in $L^{p^{\prime}}(\Omega), p^{\prime}$ is the conjugate of $p,\left(\frac{1}{p}+\frac{1}{p^{\prime}}=1\right)$

- $\Omega$ is a bounded domain in $\mathbb{R}^{N}(N \geq 2)$ which contains small inclusions $T_{\varepsilon, j}(j \in \mathbb{N})$ periodically distributed in $\Omega$ with period $\varepsilon, T_{\varepsilon, j}$ are defined by translating $\varepsilon T$, where $T$ is a closed connected regular domain inside

2000 Mathematics Subject Classification. 35B27, 35B40, 35J60.

Key words and phrases. Homogenization, perforated domain, quasilinear elliptic equations, correctors. 
the unit cube $Y=] \frac{-1}{2}, \frac{1}{2}\left[^{N}\right.$ of $\mathbb{R}^{N} . T_{\varepsilon}$ is the union of all the periodic inclusions $T_{\varepsilon, j}$.

The analysis of $\left(P_{\varepsilon}\right)$ begins by considering the associated minimization problem

$$
\min \left\{\int_{\Omega}|\nabla v|^{p} d x-p \int_{\Omega} f_{\varepsilon} v d x, v \in W_{0}^{1, p}(\Omega), v=0 \text { on } T_{\varepsilon}\right\}
$$

by standard argument, the problem $\left(D_{\varepsilon}\right)$ admits a unique solution $u_{\varepsilon}$, which is the unique weak solution of $\left(P_{\varepsilon}\right)$.

In section 2 , we give some estimates for the $L^{p}$-norms of $u_{\varepsilon}$ and $\nabla u_{\varepsilon}$ in terms of $\varepsilon$ (Proposition 2.1) and then present another equivalent version of the problem $\left(D_{\varepsilon}\right)$

$$
\min \left\{\varepsilon^{p} \int_{\Omega}|\nabla v|^{p} d x-p \int_{\Omega} f_{\varepsilon} v d x, v \in W_{0}^{1, p}(\Omega), v=0 \text { on } T_{\varepsilon}\right\}
$$

The goal of this section is to prove the epi-convergence of the problem $\left(D_{\varepsilon}^{*}\right)$ to the following problem

$$
\min \left\{\frac{1}{m(w)^{p-1}} \int_{\Omega}|v|^{p} d x-p \int_{\Omega} f v d x, v \in L^{p}(\Omega)\right\}
$$

where $w$ is the solution of the problem

$$
\begin{cases}-\Delta_{p} w=1 & \text { in } Y \backslash T \\ w=0 & \text { in } T \\ w & Y-\text { periodic }\end{cases}
$$

and $m(w)=\int_{Y} w d y$, by showing the epi-convergence of the functional

$$
F_{\varepsilon}(v)=\int_{\Omega} \varepsilon^{p}|\nabla v|^{p} d x-p \int_{\Omega} f_{\varepsilon} v d x \quad v \in W_{0}^{1, p}(\Omega), \quad v=0 \text { on } T_{\varepsilon}
$$

as $\varepsilon$ goes to zero, to the functional

$$
F(v)=\frac{1}{m(w)^{p-1}} \int_{\Omega}|v|^{p} d x-p \int_{\Omega} f v d x \quad v \in L^{p}(\Omega)
$$

that means

i) $\forall v \in L^{p}(\Omega), \exists\left(v_{\varepsilon}\right) \in W_{0}^{1, p}(\Omega), v_{\varepsilon}=0$ on $T_{\varepsilon}$ and $v_{\varepsilon} \rightarrow v \in L^{p}(\Omega)$ such that

$$
\limsup _{\varepsilon} F_{\varepsilon}\left(v_{\varepsilon}\right) \leqslant F(v)
$$

and 
ii) For all $\left(v_{\varepsilon}\right) \subseteq W_{0}^{1, p}(\Omega), v_{\varepsilon}=0$ on $T_{\varepsilon},\left(F_{\varepsilon}\left(v_{\varepsilon}\right)\right)$ bounded and $v_{\varepsilon} \rightarrow v \in L^{p}(\Omega)$ then,

$$
\liminf _{\varepsilon} F_{\varepsilon}\left(v_{\varepsilon}\right) \geqslant F(v)
$$

the proof of this property is based on Tartar's method using test functions.

For a detailed study of the concept of epi-convergence, we refer to [1].

In section 3, under some hypothesis on the sequences $\left(f_{\varepsilon}\right)$ (Theorem 3.1), we give error estimates for convergences obtained in section 2 .

The new aspects of these results are concentrated in Lemma 2.7 and Theorem 3.1. The proofs of these results are based on several vectorial inequalities given for example in [8] and [12].

For $p=2$ and $f_{\varepsilon}=f$ (for all $\varepsilon$ ), the convergence of $\left(D_{\varepsilon}\right)$ as $\varepsilon \rightarrow 0$ has been studied (see, [3], [11], [13]).

For $p \geq 2$ and $f_{\varepsilon}=f$, the limit problem of $\left(D_{\varepsilon}\right)$ as $\varepsilon \rightarrow 0$ is giving in [9].

For $f_{\varepsilon}=f$ and $p \geq 2$, the error estimates for the homogenization of $\left(D_{\varepsilon}\right)$ are given in [10].

Case $2 \leq p<\infty[2]$ (in preparation).

For a presentation of the homogenization theory see [4].

\section{Convergence of $\left(D_{\varepsilon}\right)$}

2.1. Estimates for $u_{\varepsilon}$. The weak lower semicontinuity, coercivity and strict convexity of the functional

$$
F_{\varepsilon}(v)=\int_{\Omega}|\nabla v|^{p} d x-p \int_{\Omega} f_{\varepsilon} v d x
$$

defined on the closed convex set $\left\{v \in W_{0}^{1, p}(\Omega), v=0\right.$ on $\left.T_{\varepsilon}\right\}$ of $W_{0}^{1, p}(\Omega)$ implies the existence and uniqueness of a solution $u_{\varepsilon}$ of the problem $\left(D_{\varepsilon}\right)$, that is a weak solution of $\left(P_{\varepsilon}\right)$.

Proposition 2.1. There exists a constant $c$ independent of $\varepsilon$ such that

$$
\left\|u_{\varepsilon}\right\|_{L^{p}(\Omega)} \leq c \varepsilon^{p /(p-1)} \quad \text { and }\left\|\nabla u_{\varepsilon}\right\|_{\left(L^{p}(\Omega)\right)^{N}} \leq c \varepsilon^{1 /(p-1)} .
$$

Proof. From "nonstandard" Poincaré's inequality (where $v$ in $W^{1, p}(Y \backslash T)$ and $v$ vanishes on a part of $\left.\partial(Y \backslash T)\right)$, there exists a constant $c>0$ such that, for $v \in W^{1, p}(Y \backslash T), v=0$ on $T$ we have

$$
\int_{Y \backslash T}|v|^{p} d y \leq c \int_{Y \backslash T}|\nabla v|^{p} d y .
$$

Then, for $v \in W^{1, p}(Y), v=0$ on $T$ we have

$$
\int_{Y}|v|^{p} d y \leq c \int_{Y}|\nabla v|^{p} d y .
$$


By the change of variable $y=\frac{x}{\varepsilon}$ we deduce that, for every $v_{\varepsilon}$ in $W^{1, p}(\varepsilon Y)$ such that $v_{\varepsilon}=0$ on $\varepsilon T$, we have

$$
\int_{\varepsilon Y}\left|v_{\varepsilon}\right|^{p} d x \leq c \varepsilon^{p} \int_{\varepsilon Y}\left|\nabla v_{\varepsilon}\right|^{p} d x
$$

Then, since $u_{\varepsilon}=0$ on $T_{\varepsilon}$ we have

$$
\int_{\Omega}\left|u_{\varepsilon}\right|^{p} d x \leq c \varepsilon^{p} \int_{\Omega}\left|\nabla u_{\varepsilon}\right|^{p} d x
$$

which is equivalent to

$$
\left\|u_{\varepsilon}\right\|_{L^{p}(\Omega)} \leq c \varepsilon\left\|\nabla u_{\varepsilon}\right\|_{\left(L^{p}(\Omega)\right)^{N}} .
$$

But $u_{\varepsilon}$ is the solution of $\left(D_{\varepsilon}\right)$, so

$$
\int_{\Omega}\left|\nabla u_{\varepsilon}\right|^{p} d x=\int_{\Omega} f_{\varepsilon} \cdot u_{\varepsilon} d x
$$

and we deduce that

$$
\left\|\nabla u_{\varepsilon}\right\|_{\left(L^{p}(\Omega)\right)^{N}}^{p} \leq\left\|f_{\varepsilon}\right\|_{L^{p^{\prime}}(\Omega)}\left\|u_{\varepsilon}\right\|_{L^{p}(\Omega)} \leq c\left\|u_{\varepsilon}\right\|_{L^{p}(\Omega)}
$$

(because the sequence $\left(f_{\varepsilon}\right)$ is bounded in $L^{p^{\prime}}(\Omega)$ ). It follows that

$$
\left\|u_{\varepsilon}\right\|_{L^{p}(\Omega)}^{p} \leq c \varepsilon^{p}\left\|u_{\varepsilon}\right\|_{L^{p}(\Omega)},
$$

SO

$$
\left\|u_{\varepsilon}\right\|_{L^{p}(\Omega)} \leq c \varepsilon^{p /(p-1)}
$$

and

$$
\left\|\nabla u_{\varepsilon}\right\|_{\left(L^{p}(\Omega)\right)^{N}} \leq c \varepsilon^{1 /(p-1)}
$$

where $c$ is a constant.

REMARK 2.2. We deduce from Proposition 2.1 that $\left(u_{\varepsilon}\right)$ is bounded in $W_{0}^{1, p}(\Omega)$ and then admits a converging subsequence, still denoted by $\left(u_{\varepsilon}\right)$.

REMARK 2.3. Let $u_{\varepsilon}^{*}=\varepsilon^{-p /(p-1)} u_{\varepsilon}$. Since $u_{\varepsilon}$ is the solution of $\left(D_{\varepsilon}\right), u_{\varepsilon}^{*}$ is the solution of the following minimization problem

$\left(D_{\varepsilon}^{*}\right) \quad \min \left\{\varepsilon^{p} \int_{\Omega}|\nabla v|^{p} d x-p \int_{\Omega} f_{\varepsilon} v d x \quad v \in W_{0}^{1, p}(\Omega), v=0\right.$ on $\left.T_{\varepsilon}\right\}$. 
2.2. Convergence of $\left(D_{\varepsilon}^{*}\right)$.

ThEOREM 2.4. The problem $\left(D_{\varepsilon}^{*}\right)$ converge as $\varepsilon \rightarrow 0$ to the following problem

$$
\min \left\{\frac{1}{m(w)^{p-1}} \int_{\Omega}|v|^{p} d x-p \int_{\Omega} f v d x, v \in L^{p}(\Omega)\right\}
$$

where $w$ is the solution of the problem

$$
\begin{cases}-\Delta_{p} w=1 & \text { in } Y \backslash T \\ w=0 & \text { in } T \\ w & Y-\text { periodic }\end{cases}
$$

and $m(w)=\int_{Y} w d y$. Hence,

i) $u_{\varepsilon}^{*} \rightarrow u^{*}=m(w)|f|^{p^{\prime}-2} f \quad$ in $L^{p}(\Omega)$,

ii) $\varepsilon^{\frac{-1}{(p-1)}} u_{\varepsilon} \rightarrow 0 \quad$ in $W^{1, p}(\Omega)$,

iii) $\int_{\Omega} \varepsilon^{-p /(p-1)}\left|\nabla u_{\varepsilon}\right|^{p} d x \longrightarrow m(w) \int_{\Omega}|f|^{p^{\prime}} d x$.

For proof of this theorem we use the following lemmas giving in $[10,2]$. From properties of the epi-convergence notion (see $[1,5,6,7]$ ), we get the limit problem of $\left(D_{\varepsilon}^{*}\right)$ by using a direct proof making use of suitable test functions.

LEMMA 2.5. Let $w_{\varepsilon}$ be such that

$$
\left\{\begin{array}{ll}
w_{\varepsilon}(x)=w\left(\frac{x}{\varepsilon}\right), & \text { for } x \in \varepsilon Y \backslash \varepsilon T \\
w_{\varepsilon}=0, & \text { on } \varepsilon T \\
w_{\varepsilon}, & \varepsilon Y-\text { periodic }
\end{array} .\right.
$$

Then we have

$$
\begin{cases}w_{\varepsilon} \in W^{1, p}(\Omega), w_{\varepsilon}=0, & \text { on } T_{\varepsilon}, \\ w_{\varepsilon} \rightarrow m(w), & \text { in } L^{p}(\Omega), \\ \varepsilon^{p}\left|\nabla w_{\varepsilon}\right|^{p} d x \rightarrow m(w) d x, & \text { in } \sigma\left(M_{1}(\Omega), C(\bar{\Omega})\right) .\end{cases}
$$


the following

LEMma 2.6. For all $v$ in $D(\Omega)$ the sequence $\left(v_{\varepsilon}\right)=\left(\frac{1}{m(w)} w_{\varepsilon} v\right)$ satisfies

$$
\left\{\begin{array}{l}
v_{\varepsilon} \in W_{0}^{1, p}(\Omega), \quad v_{\varepsilon}=0 \text { on } T_{\varepsilon} \\
v_{\varepsilon} \rightarrow v \text { in } L^{p}(\Omega) \\
\int_{\Omega} \varepsilon^{p}\left|\nabla v_{\varepsilon}\right|^{p} d x \rightarrow \frac{1}{m(w)^{p-1}} \int_{\Omega}|v|^{p} d x .
\end{array}\right.
$$

LEMMA 2.7. Let $v \in L^{p}(\Omega)$ and $v_{\varepsilon} \in W_{0}^{1, p}(\Omega)$ such that

$$
\begin{cases}v_{\varepsilon}=0 & \text { on } T_{\varepsilon} \\ v_{\varepsilon} \rightarrow v & \text { in } L^{p}(\Omega) \\ \left(\varepsilon\left|\nabla v_{\varepsilon}\right|\right) & \text { is bounded in } L^{p}(\Omega)\end{cases}
$$

then we have

$$
\liminf _{\varepsilon} \int_{\Omega} \varepsilon^{p}\left|\nabla v_{\varepsilon}\right|^{p} d x \geq \frac{1}{m(w)^{p-1}} \int_{\Omega}|v|^{p} d x
$$

Proof. Let $\varphi \in D(\Omega)$ and $\varphi_{\varepsilon}=\frac{1}{m(w)} \varphi w_{\varepsilon}$. By using inequality for convex functions we have

$$
\int_{\Omega} \varepsilon^{p}\left|\nabla v_{\varepsilon}\right|^{p} d x \geq \int_{\Omega} \varepsilon^{p}\left|\nabla \varphi_{\varepsilon}\right|^{p} d x+p \int_{\Omega}\left|\varepsilon \nabla \varphi_{\varepsilon}\right|^{p-2} \varepsilon \nabla \varphi_{\varepsilon} \varepsilon \nabla\left(v_{\varepsilon}-\varphi_{\varepsilon}\right) d x .
$$

But,

$$
\begin{aligned}
\int_{\Omega}\left|\varepsilon \nabla \varphi_{\varepsilon}\right|^{p-2} \varepsilon \nabla \varphi_{\varepsilon} \varepsilon \nabla\left(v_{\varepsilon}-\varphi_{\varepsilon}\right) d x & \\
& =\frac{1}{m(w)^{p-1}} \int_{\Omega}\left|\varepsilon \nabla\left(\varphi w_{\varepsilon}\right)\right|^{p-2} \varepsilon \nabla\left(\varphi w_{\varepsilon}\right) \varepsilon \nabla\left(v_{\varepsilon}-\varphi_{\varepsilon}\right) d x
\end{aligned}
$$

by using (3.5) of Lemma 3.1 given in [12] we obtain known inequality for $1<p \leq 2$

(I) $\exists \alpha>0 \quad \forall X, Y \in \mathbb{R}^{N} \quad|X|^{p-2} X-|Y|^{p-2} Y|\leq \alpha| X-\left.Y\right|^{p-1}$ 
and then,

$$
\begin{aligned}
& \left.\left|\int_{\Omega}\right| \varepsilon \nabla\left(\varphi w_{\varepsilon}\right)\right|^{p-2} \varepsilon \nabla\left(\varphi w_{\varepsilon}\right) \varepsilon \nabla\left(v_{\varepsilon}-\varphi_{\varepsilon}\right) d x \\
& -\int_{\Omega}\left|\varepsilon \varphi \nabla w_{\varepsilon}\right|^{p-2} \varepsilon \varphi \nabla w_{\varepsilon} \varepsilon \nabla\left(v_{\varepsilon}-\varphi_{\varepsilon}\right) d x \mid \\
& \leq \alpha \int_{\Omega}\left|\varepsilon w_{\varepsilon} \nabla \varphi\right|^{p-1}\left|\varepsilon \nabla\left(v_{\varepsilon}-\varphi_{\varepsilon}\right)\right| d x .
\end{aligned}
$$

Since $\varepsilon w_{\varepsilon} \rightarrow 0$ (by Lemma 2.5) and $\left(\varepsilon \nabla \varphi_{\varepsilon}\right),\left(\varepsilon \nabla v_{\varepsilon}\right)$ bounded in $L^{p}(\Omega)$ (by Lemma 2.6), we have

$$
\begin{aligned}
& \liminf _{\varepsilon} \int_{\Omega}\left|\varepsilon \nabla \varphi_{\varepsilon}\right|^{p-2} \varepsilon \nabla \varphi_{\varepsilon} \varepsilon \nabla\left(v_{\varepsilon}-\varphi_{\varepsilon}\right) d x \\
& \quad=\frac{1}{m(w)^{p-1}} \liminf _{\varepsilon} \int_{\Omega}\left|\varepsilon \varphi \nabla w_{\varepsilon}\right|^{p-2} \varepsilon \varphi \nabla w_{\varepsilon} \varepsilon \nabla\left(v_{\varepsilon}-\varphi_{\varepsilon}\right) d x .
\end{aligned}
$$

Introducing the following function

$$
\mathcal{T}_{\varepsilon}(s)= \begin{cases}|s|^{p-2} s, & \text { if }|s|>\varepsilon \\ |\varepsilon|^{p-2} s, & \text { if }|s| \leq \varepsilon\end{cases}
$$

we have

$$
\begin{aligned}
\int_{\Omega} \mid \varepsilon \varphi & \left.\nabla w_{\varepsilon}\right|^{p-2} \varepsilon \varphi \nabla w_{\varepsilon} \varepsilon \nabla\left(v_{\varepsilon}-\varphi_{\varepsilon}\right) d x \\
= & \int_{\Omega} \varepsilon^{p}\left|\nabla w_{\varepsilon}\right|^{p-2} \mathcal{T}_{\varepsilon}(\varphi) \nabla w_{\varepsilon} \nabla\left(v_{\varepsilon}-\varphi_{\varepsilon}\right) d x \\
& +\int_{\{x \in \Omega:|\varphi| \leq \varepsilon\}} \varepsilon^{p}\left|\nabla w_{\varepsilon}\right|^{p-2}\left(|\varphi|^{p-2} \varphi-\mathcal{T}_{\varepsilon}(\varphi)\right) \nabla w_{\varepsilon} \nabla\left(v_{\varepsilon}-\varphi_{\varepsilon}\right) d x
\end{aligned}
$$

But,

$$
\begin{aligned}
& \left.\left|\int_{\{x \in \Omega:|\varphi| \leq \varepsilon\}} \varepsilon^{p}\right| \nabla w_{\varepsilon}\right|^{p-2}\left(|\varphi|^{p-2} \varphi-\mathcal{T}_{\varepsilon}(\varphi)\right) \nabla w_{\varepsilon} \nabla\left(v_{\varepsilon}-\varphi_{\varepsilon}\right) d x \mid \\
& \quad \leq\left. 2 \varepsilon^{p-1}\left|\int_{\Omega}\right| \varepsilon \nabla w_{\varepsilon}\right|^{p-1}\left|\varepsilon \nabla\left(v_{\varepsilon}-\varphi_{\varepsilon}\right)\right| d x \mid \\
& \quad \leq 2 \varepsilon^{p-1}\left\|\varepsilon \nabla w_{\varepsilon}\right\|_{\left(L^{p}(\Omega)\right)^{N}}^{p-1}\left\|\varepsilon \nabla\left(v_{\varepsilon}-\varphi_{\varepsilon}\right)\right\|_{\left(L^{p}(\Omega)\right)^{N}}
\end{aligned}
$$


(from Hölder's inequality). Since the sequences $\left(\left|\varepsilon \nabla w_{\varepsilon}\right|\right)$ and $\left(\left|\varepsilon \nabla\left(v_{\varepsilon}-\varphi_{\varepsilon}\right)\right|\right)$ are bounded in $L^{p}(\Omega)$ (due to Lemmas 2.5 and 2.6) we deduce,

$$
\begin{aligned}
& \liminf _{\varepsilon} \int_{\Omega} \varepsilon^{p}\left|\varphi \nabla w_{\varepsilon}\right|^{p-2} \varphi \nabla w_{\varepsilon} \nabla\left(v_{\varepsilon}-\varphi_{\varepsilon}\right) d x \\
& \quad=\liminf _{\varepsilon} \int_{\Omega} \varepsilon^{p}\left|\nabla w_{\varepsilon}\right|^{p-2} \nabla w_{\varepsilon} \mathcal{T}_{\varepsilon}(\varphi) \nabla\left(v_{\varepsilon}-\varphi_{\varepsilon}\right) d x .
\end{aligned}
$$

Now,

$$
\begin{aligned}
\int_{\Omega} \varepsilon^{p}\left|\nabla w_{\varepsilon}\right|^{p-2} \nabla w_{\varepsilon} \mathcal{T}_{\varepsilon}(\varphi) \nabla\left(v_{\varepsilon}-\varphi_{\varepsilon}\right) d x \\
=\int_{\Omega} \varepsilon^{p}\left|\nabla w_{\varepsilon}\right|^{p-2} \nabla w_{\varepsilon} \nabla\left(\mathcal{T}_{\varepsilon}(\varphi)\left(v_{\varepsilon}-\varphi_{\varepsilon}\right)\right) d x \\
\quad-\int_{\Omega} \varepsilon^{p}\left|\nabla w_{\varepsilon}\right|^{p-2} \nabla w_{\varepsilon} \nabla\left(\mathcal{T}_{\varepsilon}(\varphi)\right) \cdot\left(v_{\varepsilon}-\varphi_{\varepsilon}\right) d x
\end{aligned}
$$

and

$$
\begin{aligned}
& \left.\left|\int_{\Omega} \varepsilon^{p}\right| \nabla w_{\varepsilon}\right|^{p-2} \nabla w_{\varepsilon} \nabla\left(\mathcal{T}_{\varepsilon}(\varphi)\right) \cdot\left(v_{\varepsilon}-\varphi_{\varepsilon}\right) d x \mid \\
& \quad \leq c \varepsilon^{p-2} \int_{\Omega} \varepsilon^{p}\left|\nabla w_{\varepsilon}\right|^{p-1}\left|\left(v_{\varepsilon}-\varphi_{\varepsilon}\right)\right| d x \\
& \quad \leq c \varepsilon^{p-1}\left\|\varepsilon \nabla w_{\varepsilon}\right\|_{\left(L^{p}(\Omega)\right)^{N}}^{p-1}\left\|v_{\varepsilon}-\varphi_{\varepsilon}\right\|_{L^{p}(\Omega)}
\end{aligned}
$$

(by using again Hölder's inequality). Since the sequences $\left(\left|\left(\varepsilon \nabla w_{\varepsilon}\right)\right|\right)$ and $\left(\left|v_{\varepsilon}-\varphi_{\varepsilon}\right|\right)$ are bounded in $L^{p}(\Omega)$, we deduce again that

$$
\begin{aligned}
& \liminf _{\varepsilon} \int_{\Omega} \varepsilon^{p}\left|\varphi \nabla w_{\varepsilon}\right|^{p-2} \varphi \nabla w_{\varepsilon} \nabla\left(v_{\varepsilon}-\varphi_{\varepsilon}\right) d x \\
& \quad=\liminf _{\varepsilon} \int_{\Omega} \varepsilon^{p}\left|\nabla w_{\varepsilon}\right|^{p-2} \nabla w_{\varepsilon} \nabla\left(\mathcal{T}_{\varepsilon}(\varphi)\left(v_{\varepsilon}-\varphi_{\varepsilon}\right)\right) d x \\
& \quad=\liminf _{\varepsilon} \int_{\Omega} \mathcal{T}_{\varepsilon}(\varphi)\left(v_{\varepsilon}-\varphi_{\varepsilon}\right) d x
\end{aligned}
$$


(by the definition of $w_{\varepsilon}$ ). Since

$$
\begin{aligned}
\int_{\Omega} \mathcal{T}_{\varepsilon}(\varphi)\left(v_{\varepsilon}-\varphi_{\varepsilon}\right) d x= & \int_{\Omega}|\varphi|^{p-2} \varphi\left(v_{\varepsilon}-\varphi_{\varepsilon}\right) d x \\
& +\int_{\{x \in \Omega:|\varphi| \leq \varepsilon\}}\left(|\varepsilon|^{p-2} \varphi-|\varphi|^{p-2} \varphi\right)\left(v_{\varepsilon}-\varphi_{\varepsilon}\right) d x
\end{aligned}
$$

and (from Hölder's inequality)

$$
\left|\int_{\{x \in \Omega:|\varphi| \leq \varepsilon\}}\left(|\varepsilon|^{p-2} \varphi-|\varphi|^{p-2} \varphi\right)\left(v_{\varepsilon}-\varphi_{\varepsilon}\right) d x\right| \leq c \varepsilon^{p-1}\left\|v_{\varepsilon}-\varphi_{\varepsilon}\right\|_{L^{p}(\Omega)},
$$

it follows that

$$
\begin{aligned}
\liminf _{\varepsilon} \int_{\Omega} \varepsilon^{p}\left|\varphi \nabla w_{\varepsilon}\right|^{p-2} \varphi \nabla w_{\varepsilon} \nabla\left(v_{\varepsilon}-\varphi_{\varepsilon}\right) d x & =\liminf _{\varepsilon} \int_{\Omega}|\varphi|^{p-2} \varphi\left(v_{\varepsilon}-\varphi_{\varepsilon}\right) d x \\
& =\int_{\Omega}|\varphi|^{p-2} \varphi(v-\varphi) d x
\end{aligned}
$$

(because $v_{\varepsilon} \rightarrow v$ and $\varphi_{\varepsilon} \rightarrow \varphi$ in $L^{p}(\Omega)$ ). Finally,

$\liminf _{\varepsilon} \int_{\Omega} \varepsilon^{p}\left|\nabla v_{\varepsilon}\right|^{p} d x \geq \liminf _{\varepsilon} \int_{\Omega} \varepsilon^{p}\left|\nabla \varphi_{\varepsilon}\right|^{p} d x+\frac{p}{m(w)^{p-1}} \int_{\Omega}|\varphi|^{p-2} \varphi(v-\varphi) d x$ and (from Lemma 2.6)

$\liminf _{\varepsilon} \int_{\Omega} \varepsilon^{p}\left|\nabla v_{\varepsilon}\right|^{p} d x \geq \frac{1}{m(w)^{p-1}} \int_{\Omega}|\varphi|^{p} d x+\frac{p}{m(w)^{p-1}} \int_{\Omega}|\varphi|^{p-2} \varphi(v-\varphi) d x$

by choosing $\varphi$ converges to $v$ in $s-L^{p}(\Omega)$, we obtain

$$
\liminf _{\varepsilon} \int_{\Omega} \varepsilon^{p}\left|\nabla v_{\varepsilon}\right|^{p} d x \geq \frac{1}{m(w)^{p-1}} \int_{\Omega}|v|^{p} d x .
$$

Proof of Theorem 2.4. Let $v \in D(\Omega)$. By Lemma 2.6 there exist $v_{\varepsilon}$ in $W_{0}^{1, p}(\Omega)$ such that

$$
\left\{\begin{array}{l}
v_{\varepsilon}=0 \text { on } T_{\varepsilon}, \\
v_{\varepsilon} \rightarrow v \text { in } L^{p}(\Omega), \\
\int_{\Omega} \varepsilon^{p}\left|\nabla v_{\varepsilon}\right|^{p} d x \rightarrow \frac{1}{m(w)^{p-1}} \int_{\Omega}|v|^{p} d x .
\end{array}\right.
$$

Now, by Proposition 2.1, the sequence $\left(u_{\varepsilon}^{*}\right)$ is bounded in $L^{p}(\Omega)$ and admits a weakly converging subsequence in $L^{p}(\Omega)$ still denoted by $\left(u_{\varepsilon}^{*}\right)$. 
Let $u^{*}=\lim _{\varepsilon \rightarrow 0} u_{\varepsilon}^{*}$ in $w-L^{p}(\Omega)$. Since

$$
\liminf _{\varepsilon} \int_{\Omega} \varepsilon^{p}\left|\nabla u_{\varepsilon}^{*}\right|^{p} d x \geq \frac{1}{m(w)^{p-1}} \int_{\Omega}\left|u^{*}\right|^{p} d x \quad \text { (from Lemma 2.7) }
$$

we have

$$
\begin{aligned}
& \frac{1}{m(w)^{p-1}} \int_{\Omega}\left|u^{*}\right|^{p} d x-p \int_{\Omega} f u^{*} d x \\
& \quad \leq \liminf _{\varepsilon}\left(\int_{\Omega} \varepsilon^{p}\left|\nabla u_{\varepsilon}^{*}\right|^{p} d x-p \int_{\Omega} f_{\varepsilon} u_{\varepsilon}^{*} d x\right) \\
& \quad \leq \liminf _{\varepsilon}\left(\int_{\Omega} \varepsilon^{p}\left|\nabla v_{\varepsilon}^{*}\right|^{p} d x-p \int_{\Omega} f_{\varepsilon} v_{\varepsilon}^{*} d x\right) \quad \text { (by the definition of } u_{\varepsilon}^{*} \text { ) } \\
& \quad \leq \frac{1}{m(w)^{p-1}} \int_{\Omega}|v|^{p} d x-p \int_{\Omega} f v d x
\end{aligned}
$$

(by Lemma 2.6 and this for all $v \in D(\Omega)$ ). Then by the density of $D(\Omega)$ in $L^{p}(\Omega)$ for all $v$ in $L^{p}(\Omega)$ we have

$$
\frac{1}{m(w)^{p-1}} \int_{\Omega}\left|u^{*}\right|^{p} d x-p \int_{\Omega} f u^{*} d x \leq \frac{1}{m(w)^{p-1}} \int_{\Omega}|v|^{p} d x-p \int_{\Omega} f v d x
$$

which is equivalent to: $u^{*}$ is the solution of the minimization problem $\left(D^{*}\right)$. But the unique solution of the problem $\left(D^{*}\right)$ is $m(w)|f|^{p^{\prime}-2} f$ then $u^{*}=$ $m(w)|f|^{p^{\prime}-2} f$.

\section{Error estimates}

In this paragraph, we establish error estimates for the convergence obtained in Theorem 2.4. that

TheOrem 3.1. Assume that $\left(f_{\varepsilon}\right)$ is a bounded sequence in $W_{0}^{1, \infty}(\Omega)$ such

$$
\left\|f_{\varepsilon}-f\right\|_{\infty} \leq c \varepsilon^{r} \quad(r>0) .
$$

Then we have

I) $\left\|\frac{u_{\varepsilon}}{\varepsilon^{p^{\prime}}}-w_{\varepsilon}|f|^{p^{\prime}-2} f\right\|_{L^{p}(\Omega)} \leq\left\{\begin{array}{ll}c \varepsilon^{r(p-1)}, & \text { if } 0<r<1 \\ c \varepsilon^{p-1}, & \text { if } r \geq 1\end{array}\right.$,

II) $\left.\left|\int_{\Omega} \frac{\left|\nabla u_{\varepsilon}\right|^{p}}{\varepsilon^{p^{\prime}}} d x-\int_{\Omega} w_{\varepsilon}\right| f\right|^{p^{\prime}} d x \mid \leq\left\{\begin{array}{ll}c \varepsilon^{r(p-1)}, & \text { if } 0<r<1 \\ c \varepsilon^{p-1}, & \text { if } \quad r \geq 1\end{array}\right.$. 
Proof OF I). Let $v_{\varepsilon}=\frac{u_{\varepsilon}}{\varepsilon^{p^{\prime}}}-w_{\varepsilon} g$ with $g=|f|^{p^{\prime}-2} f$. Since $v_{\varepsilon}=0$ on $T_{\varepsilon}$ we have

$$
\left\|v_{\varepsilon}\right\|_{L^{p}(\Omega)} \leq c \varepsilon\left\|\nabla v_{\varepsilon}\right\|_{\left(L^{p}(\Omega)\right)^{N}}
$$

(see proof of Proposition 2.1). Let us estimate $\left\|\nabla v_{\varepsilon}\right\|_{\left(L^{p}(\Omega)\right)^{N}}$ :

Using the known inequality for $1<p \leq 2$ (see for example [12, Lemma 3.1])

$$
\exists c>0 \quad|X-Y|^{2} \leq c(|X|+|Y|)^{2-p}\left(|X|^{p-2} X-|Y|^{p-2} Y\right)(X-Y)
$$

for all $X, Y \in \mathbb{R}^{N}$ such that $|X|+|Y| \neq 0$, we obtain

$$
\begin{aligned}
\int_{\Omega}\left|\nabla v_{\varepsilon}\right|^{p} d x= & \int_{\Omega}\left|\nabla\left(\frac{u_{\varepsilon}}{\varepsilon^{p^{\prime}}}\right)-\nabla\left(w_{\varepsilon} g\right)\right|^{p} d x \\
\leq & c \int_{\Omega}\left(\left|\nabla\left(\frac{u_{\varepsilon}}{\varepsilon^{p^{\prime}}}\right)\right|+\left|\nabla\left(w_{\varepsilon} g\right)\right|\right)^{\frac{p(2-p)}{2}}\left[\left(\left|\nabla\left(\frac{u_{\varepsilon}}{\varepsilon p^{\prime}}\right)\right|^{p-2} \nabla\left(\frac{u_{\varepsilon}}{\varepsilon^{p^{\prime}}}\right)\right.\right. \\
& \left.\left.\quad-\left|\nabla\left(w_{\varepsilon} g\right)\right|^{p-2} \nabla\left(w_{\varepsilon} g\right)\right) \nabla v_{\varepsilon}\right]^{\frac{p}{2}} d x .
\end{aligned}
$$

Then

$$
\begin{aligned}
\int_{\Omega}\left|\nabla v_{\varepsilon}\right|^{p} d x \leq & c M_{\varepsilon}\left[\int_{\Omega}\left|\nabla\left(\frac{u_{\varepsilon}}{\varepsilon^{p^{\prime}}}\right)\right|^{p-2} \nabla\left(\frac{u_{\varepsilon}}{\varepsilon^{p^{\prime}}}\right) \nabla v_{\varepsilon} d x\right. \\
& \left.-\int_{\Omega}\left|\nabla\left(w_{\varepsilon} g\right)\right|^{p-2} \nabla\left(w_{\varepsilon} g\right) \nabla v_{\varepsilon} d x\right]^{\frac{p}{2}}
\end{aligned}
$$

(by using Hölder's inequality) and where

$$
M_{\varepsilon}=\left[\int_{\Omega}\left(\left|\nabla\left(\frac{u_{\varepsilon}}{\varepsilon^{p^{p^{\prime}}}}\right)\right|+\left|\nabla\left(w_{\varepsilon} g\right)\right|\right)^{p} d x\right]^{\frac{(2-p)}{2}} .
$$

But

$$
\begin{aligned}
M_{\varepsilon} & \leq c\left[\int_{\Omega}\left(\left|\nabla\left(\frac{u_{\varepsilon}}{\varepsilon^{p^{\prime}}}\right)\right|^{p}+\left|\nabla\left(w_{\varepsilon} g\right)\right|^{p}\right) d x\right]^{\frac{(2-p)}{2}} \\
& \leq c\left[\int_{\Omega}\left(\left|\nabla\left(\frac{u_{\varepsilon}}{\varepsilon^{p^{\prime}}}\right)\right|^{p}+\left|w_{\varepsilon}\right|^{p}+\left|\nabla w_{\varepsilon}\right|^{p}\right) d x\right]^{\frac{(2-p)}{2}} \\
& \leq \frac{c}{\varepsilon^{\frac{p(2-p)}{2}}} \quad \text { (by using Proposition 2.1 and Lemma 2.5). }
\end{aligned}
$$


By definitions of $u_{\varepsilon}$ and $w_{\varepsilon}$ we have

$$
\begin{aligned}
\int_{\Omega}\left|\nabla\left(\frac{u_{\varepsilon}}{\varepsilon^{p^{\prime}}}\right)\right|^{p-2} \nabla\left(\frac{u_{\varepsilon}}{\varepsilon^{p^{\prime}}}\right) \nabla v_{\varepsilon} d x & =\frac{1}{\varepsilon^{p}} \int_{\Omega} f_{\varepsilon} v_{\varepsilon} d x \\
& =\int_{\Omega}\left|\nabla w_{\varepsilon}\right|^{p-2} \nabla w_{\varepsilon} \nabla\left(f_{\varepsilon} v_{\varepsilon}\right) d x
\end{aligned}
$$

and then

$$
\begin{aligned}
& \int_{\Omega}\left|\nabla v_{\varepsilon}\right|^{p} d x \leq c M_{\varepsilon}\left[\int_{\Omega}\left|\nabla w_{\varepsilon}\right|^{p-2} \nabla w_{\varepsilon} f_{\varepsilon} \nabla v_{\varepsilon} d x\right. \\
& \left.\quad+\int_{\Omega}\left|\nabla w_{\varepsilon}\right|^{p-2} \nabla w_{\varepsilon} v_{\varepsilon} \nabla f_{\varepsilon} d x-\int_{\Omega}\left|\nabla\left(w_{\varepsilon} g\right)\right|^{p-2} \nabla\left(w_{\varepsilon} g\right) \nabla v_{\varepsilon} d x\right]^{\frac{p}{2}} .
\end{aligned}
$$

Since (by Hölder's inequality, by Lemma 2.5 and Proposition 2.1)

$$
\begin{aligned}
\int_{\Omega}\left|\nabla w_{\varepsilon}\right|^{p-2} \nabla w_{\varepsilon} v_{\varepsilon} \nabla f_{\varepsilon} d x & \leq c\left\|v_{\varepsilon}\right\|_{L^{p}(\Omega)}\left\|\nabla w_{\varepsilon}\right\|_{\left(L^{p}(\Omega)\right)^{N}}^{p-1} \\
& \leq \frac{c}{\varepsilon^{p-2}}\left\|\nabla v_{\varepsilon}\right\|_{\left(L^{p}(\Omega)\right)^{N}}
\end{aligned}
$$

and

$$
\begin{aligned}
\left.\int_{\Omega}\left|\nabla w_{\varepsilon}\right|\right|^{p-2} \nabla w_{\varepsilon} f_{\varepsilon} \nabla v_{\varepsilon} d x-\int_{\Omega}\left|\nabla\left(w_{\varepsilon} g\right)\right|^{p-2} \nabla\left(w_{\varepsilon} g\right) \nabla v_{\varepsilon} d x \\
=\int_{\Omega}\left|g_{\varepsilon} \nabla w_{\varepsilon}\right|^{p-2} g_{\varepsilon} \nabla w_{\varepsilon} \nabla v_{\varepsilon} d x-\int_{\Omega}\left|\nabla\left(w_{\varepsilon} g\right)\right|^{p-2} \nabla\left(w_{\varepsilon} g\right) \nabla v_{\varepsilon} d x
\end{aligned}
$$

(where $g_{\varepsilon}=\left|f_{\varepsilon}\right|^{p^{\prime}-2} f_{\varepsilon}$ ) and

$$
\begin{aligned}
\left.\int_{\Omega}\left|g_{\varepsilon} \nabla w_{\varepsilon}\right|\right|^{p-2} g_{\varepsilon} \nabla w_{\varepsilon} \nabla v_{\varepsilon} d x-\int_{\Omega}\left|\nabla\left(w_{\varepsilon} g\right)\right|^{p-2} \nabla\left(w_{\varepsilon} g\right) \nabla v_{\varepsilon} d x \\
=\int_{\Omega}\left|g_{\varepsilon} \nabla w_{\varepsilon}\right|^{p-2} g_{\varepsilon} \nabla w_{\varepsilon} \nabla v_{\varepsilon} d x-\int_{\Omega}\left|g \nabla w_{\varepsilon}\right|^{p-2} g \nabla w_{\varepsilon} \nabla v_{\varepsilon} d x \\
\quad+\int_{\Omega}\left|g \nabla w_{\varepsilon}\right|^{p-2} g \nabla w_{\varepsilon} \nabla v_{\varepsilon} d x-\int_{\Omega}\left|\nabla\left(w_{\varepsilon} g\right)\right|^{p-2} \nabla\left(w_{\varepsilon} g\right) \nabla v_{\varepsilon} d x \\
\leq c\left(\int_{\Omega}\left|\left(g_{\varepsilon}-g\right) \nabla w_{\varepsilon}\right|^{p-1}\left|\nabla v_{\varepsilon}\right| d x+\int_{\Omega}\left|w_{\varepsilon} \nabla g\right|^{p-1}\left|\nabla v_{\varepsilon}\right| d x\right)
\end{aligned}
$$


(we obtain latest inequality by using again inequality (I))

$$
\leq c\left(\varepsilon^{r(p-1)} \int_{\Omega}\left|\nabla w_{\varepsilon}\right|^{p-1}\left|\nabla v_{\varepsilon}\right| d x+\int_{\Omega}\left|w_{\varepsilon}\right|^{p-1}\left|\nabla v_{\varepsilon}\right| d x\right)
$$

(because $\left\|f_{\varepsilon}-f\right\|_{\infty} \leq c \varepsilon^{r}$ then $\left\|g_{\varepsilon}-g\right\|_{\infty} \leq c \varepsilon^{r}$ too, and this from known inequality for $p^{\prime} \geq 2$ (see for example Lemma 3.1 in [12])

$$
\left.\forall X, Y \in \mathbb{R}^{N}, \exists c>\left.0|| X\right|^{p^{\prime}-2} X-|Y|^{p^{\prime}-2} Y|\leq c| X-Y \mid(|X|+|Y|)^{p^{\prime}-2}\right)
$$

then

$$
\begin{aligned}
& \int_{\Omega}\left|\nabla w_{\varepsilon}\right|^{p-2} \nabla w_{\varepsilon} f_{\varepsilon} \nabla v_{\varepsilon} d x-\int_{\Omega}\left|\nabla\left(w_{\varepsilon} g\right)\right|^{p-2} \nabla\left(w_{\varepsilon} g\right) \nabla v_{\varepsilon} d x \\
& \quad \leq c\left(\varepsilon^{r(p-1)}\left\|\nabla w_{\varepsilon}\right\|_{\left(L^{p}(\Omega)\right)^{N}}^{p-1}\left\|\nabla v_{\varepsilon}\right\|_{\left(L^{p}(\Omega)\right)^{N}}+\left\|w_{\varepsilon}\right\|_{L^{p}(\Omega)}^{p-1}\left\|\nabla v_{\varepsilon}\right\|_{\left(L^{p}(\Omega)\right)^{N}}\right) \\
& \quad \leq c\left(\frac{\varepsilon^{r(p-1)}}{\varepsilon^{p-1}}+1\right)\left\|\nabla v_{\varepsilon}\right\|_{\left(L^{p}(\Omega)\right)^{N}}
\end{aligned}
$$

(by using again the Lemma 2.5). It follows that

$$
\begin{aligned}
\int_{\Omega}\left|\nabla v_{\varepsilon}\right|^{p} d x & \leq \frac{c}{\varepsilon^{\frac{p(2-p)}{2}}}\left[\left(\frac{1}{\varepsilon^{p-2}}+\frac{\varepsilon^{r(p-1)}}{\varepsilon^{p-1}}+1\right)\left\|\nabla v_{\varepsilon}\right\|_{\left(L^{p}(\Omega)\right)^{N}}\right]^{\frac{p}{2}} \\
& \leq \frac{c}{\varepsilon^{\frac{p(2-p)}{2}}}\left[\left(\frac{\varepsilon^{r(p-1)}}{\varepsilon^{p-1}}+1\right)\left\|\nabla v_{\varepsilon}\right\|_{\left(L^{p}(\Omega)\right)^{N}}\right]^{\frac{p}{2}}
\end{aligned}
$$

and

Hence,

$$
\left\|\nabla v_{\varepsilon}\right\|_{\left(L^{p}(\Omega)\right)^{N}} \leq \frac{c}{\varepsilon^{2-p}}\left(\varepsilon^{(p-1)(r-1)}+1\right) .
$$

$$
\left\|v_{\varepsilon}\right\|_{L^{p}(\Omega)} \leq \frac{c}{\varepsilon^{1-p}}\left(\varepsilon^{(p-1)(r-1)}+1\right) \leq \begin{cases}c \varepsilon^{r(p-1)}, & \text { if } \quad 0<r<1 \\ c \varepsilon^{p-1}, & \text { if } \quad r \geq 1\end{cases}
$$

Proof OF II). Since

$$
\frac{1}{\varepsilon^{p^{\prime}}} \int_{\Omega}\left|\nabla u_{\varepsilon}\right|^{p} d x=\frac{1}{\varepsilon^{p^{\prime}}} \int_{\Omega} f_{\varepsilon} u_{\varepsilon} d x=\int_{\Omega} f_{\varepsilon} v_{\varepsilon} d x+\int_{\Omega} f_{\varepsilon} w_{\varepsilon} g d x
$$

then

$$
\begin{aligned}
\left.\left|\frac{1}{\varepsilon^{p^{\prime}}} \int_{\Omega}\right| \nabla u_{\varepsilon}\right|^{p} d x-\int_{\Omega} w_{\varepsilon}|f|^{p^{\prime}} d x \mid & =\left|\int_{\Omega} f_{\varepsilon} v_{\varepsilon} d x+\int_{\Omega} w_{\varepsilon}\left(f_{\varepsilon} g-|f|^{p^{\prime}}\right) d x\right| \\
& \leq c\left\|v_{\varepsilon}\right\|_{L^{p}(\Omega)}+c \varepsilon^{r},
\end{aligned}
$$

finally we obtain II) by using I). 


\section{REFERENCES}

[1] H. Attouch, Variational convergence for functions and operators, Applicable Mathematics Series, Pitman, London, 1984.

[2] L. Boukrim, A. Hakim and T. Mekkaoui, Limiting behaviour and error estimates for the Dirichlet problem with p-Laplacian in a periodically perfored domain, in preparation.

[3] D. Cioranescu and F. Murat, Un terme étrange venu d'aileurs, Collège de France Seminar, Research Notes in Mathematics, Pitman, London, 1982, No. 60, pp. 98-138, No. 70, pp. 154-178.

[4] D. Cioranescu and P. Donato, An Introduction to Homogenization, Oxford Univ. Press, 1999.

[5] G. Dal Maso, An Introduction to $\Gamma$-convergence, Birkhäuser, Boston, 1993.

[6] G. Dal Maso and P. Longo, Г-limits of ogstacles, Ann. Mat. Pura Appl. 128 (1981), $1-50$.

[7] E. De Giorgi and T. Franzoni, Su un tipo di convergenza variazionale, Rend. Acad. Naz. Lincei 58 (1975), 842-850.

[8] R. Glowinski and A. Marroco, Sur l'approximation par éléments finis d'ordre un, et la résolution, par pénalisation -dualité, d'une classe de problémes de Dirichlet non linéaires, RAIRO 9 (1975), 41-76.

[9] N. Labani and C. Picard, Homogenization of nonlinear Dirichlet problem in a perfored domain, in "Recent advances in nonlinear eleptic and parabolic problems", Pitman Res. Notes Math. Ser. 208 (1989), 296-305.

[10] T. Mekkaoui and C. Picard, Error estimates for the homogenization of a quasilinear Dirichlet problem in a periodically perfored domain, Progress in P.D.E: the Metz Survey 2, M.Chipot Ed., Pitman Res. Notes in Math. Series 296 (1993), 185-193.

[11] C. Picard, Analyse limite d'équations variationnelles dans un domaine contenant une grille, Math. Model. Numer. Anal. 21 (1987), 293-326.

[12] D. Sandri, Sur l'approximation numérique des écoulements quasi-newtoniens dont la viscosité suit la loi puissance ou la loi de carreau, Math. Model. Numer. Anal. 27 (1993), 131-155.

[13] L. Tartar, Cours Peccot au collège de France, mars 1977, non publié.

L. Boukrim

Université Moulay Ismail

F.S.T.E

BP509 Boutalamine Errachidia

Maroc

A. Hakim

Université Cadi Ayyad

F.S.T.G

BP 549 Av. Abdelkrim Khattabi Marrakech

Maroc

T. Mekkaoui

Université Moulay Ismail

F.S.T.E

BP509 Boutalamine Errachidia

Maroc

Received: 13.7.2006.

Revised: 13.11.2006. 